\title{
A New Tracking Algorithm for Strong-Maneuvering Target with Two-Layer Nested Model of CS and CL
}

\author{
Jinshuan Peng ${ }^{1}$, Lei Xu ${ }^{1}$, Liping Wang ${ }^{2}$ and Xiaoxiang Zhou ${ }^{1}$ \\ ${ }^{1}$ Chongqing Key Lab of Traffic System \& Safety in Mountain Cities, Chongqing Jiaotong \\ University, Chongqing 400074, China \\ ${ }^{2}$ School of Computer Science, Engineering and Mathematics, Flinders University, South Australia \\ 5042, Australia \\ Corresponding Author: Peng Jinshuan, pengjinshuan@163.com
}

\begin{abstract}
A new tracking algorithm is proposed for strong-maneuvering target, which is based on a two-layer nested model with Improved Current Statistical (ICS) model and Curvilinear (CL) model as the inner and outer layer respectively. The inner layer use ICS model to construct statistics with filtering residuals to detect target's maneuver and thus correcting the parameters of CS model in real time in order to adapt to target's real motion. The outer layer uses the estimate of acceleration obtained from the inner layer as its input and in this way conduct better performance of target tracking by taking advantage of CL model, which can better correspond to the curvilinear motion of target. Simulation results show the practicability of the algorithm proposed in this article and demonstrate good tracking performance.
\end{abstract}

Keywords: strong-maneuvering target; two-layer nested model; improved current statistical model; Curvilinear model; maneuver detection

\section{Introduction}

The improving maneuver ability of aerial vehicles along with the fast development of modern technology turns the motion model construction of target into a crucial as well as difficult part in the target tracking technology of modern air combat. Therefore, the design of tracking algorithms for strong-maneuver target counts a lot in conducting good tracking performance [1].

The Current Statistical (CS) model proposed by Zhou Hongren in 1983 is widely used in target tracking. However, the values of angular acceleration limits $a_{ \pm \max }$ and maneuver frequency $\alpha(k)$ are both decided empirically and fixed during the whole filtering process, which would result in considerably decrease of tracking accuracy. Improved CS (ICS) model using Activation Function constructed by filtering residuals is designed in [2-3] to correct the variance of acceleration and maneuver frequency. Curvilinear model, which contains constant velocity, constant acceleration and constant angular velocity motion models under different model parameters, is proposed in [4] to better correspond with the real motion of target.

To conduct better tracking performance, a new tracking algorithm with two-layer nested model is proposed in this article, the inner and outer layer of which are respectively ICS model and CL model. As the inner layer, ICS model uses adaptive-adjustment algorithm to estimate the acceleration of target in real time. Then the CL model of the outer layer uses this 
estimate to better approximate the curvilinear motion of target. Through the combination of CS and CL model, stable target tracking with high accuracy is realized.

\section{System Configuration of the Two-Layer Models}

In Figure $1, z(k)$ is the measurement set, $v(k)$ is the filtering residual, $\alpha(k)$ is the maneuvering frequency, $Q(k)$ is the error covariance of the acceleration, $\hat{a}_{t}(k)$ is the tangential acceleration estimate, $\hat{x}_{T}(k)$ is target state estimate.

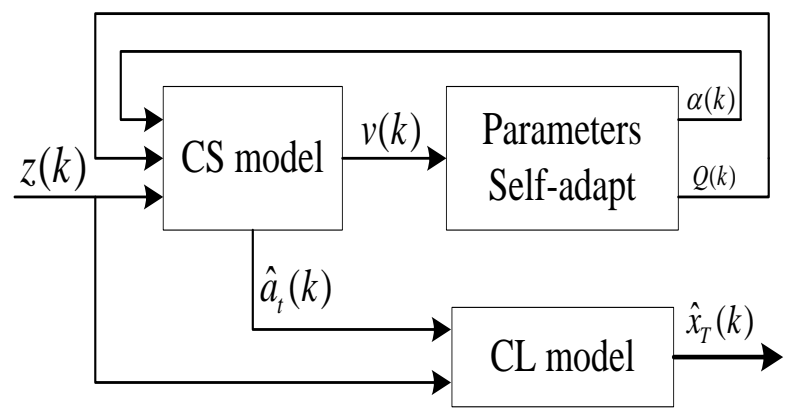

Figure 1. The Block Diagram of the Two-layer Model

The two-layer model is composed of ICS model and CL model. The inner model is Improved Current Statistical (ICS) model, and it uses the self-adaptive adjustment algorithm to adjust the parameters, so it can estimate the target acceleration with greater accuracy. The outer model is CL model which takes the acceleration estimate from ICS as on of the inputs. And because CL model has a great approximation to the target curve motion, so it can track the target precisely.

\section{Improved CS Model}

\subsection{Maneuver Detecting Algorithm of Target}

In standard CS model, the maneuvering frequency $(\alpha(k))$ and the minimum and the maximum accelerate $\left(a_{ \pm \max }\right)$ of target can directly influence the state transition matrix $(F(k))$, turbulence transfer matrix $(U(k))$ and the variance of acceleration error $\left(_{Q(k)}\right)$ of the CS model, thus changing the target tracking accuracy. The values of $\alpha(k)$ and $a_{ \pm \max }$ in standard CS model are usually fixed and empirical, which can conduct good tracking performance only when the values happen to be in accord with the target's real maneuver, which is often supposed to be week. However, as the vehicles used in modern combat are generally good at maneuvering, it is obviously that fixed model parameters can't conduct tracking performance that is satisfactory enough.

Based on that the statistical characters of filtering residual would change when target maneuvers, we designed a maneuver detecting algorithm of target.

The filtering residual in UKF is as following:

$$
v(k)=Z(k)-\bar{Z}(k / k-1)
$$


Considering that $v(k)$ is a White Gaussian when there's no maneuver, the variance matrix of $v(k)$ is as follows:

$$
\begin{aligned}
S(k)=\sum_{l=0}^{2 n}\left\{w _ { l } ^ { ( c ) } \left[\left(\mathrm{z}^{l}(k+1 / k)-\bar{Z}^{l}(k+1 / k)\right) \square\right.\right. \\
\\
\left.\left.\left(\mathrm{Z}^{l}(k+1 / k)-\bar{Z}^{l}(k+1 / k)\right)^{T}\right]\right\}+R(k+1)
\end{aligned}
$$

Construct a statistical scale $d(k)$ as:

$$
d(k)=v(k)^{T} \square S(k)^{-1} \square_{v}(k)
$$

From the statistical characters of filtering residual, we know that:

$$
d(k) \sim \chi^{2}(m)
$$

Where $m$ stands for observation dimension. While $v(k)$ is no longer a white Gaussian when the target begins to maneuver. Moreover, the stronger the target's maneuver is, the larger $d(k)$ becomes. The maneuver detecting algorithm designed based on this character is as following.

Set a false alarm probability as $\lambda_{T a}$, then the corresponding threshold $T a$ can be got by reference of distribution table of $\chi^{2}(m)$.

$$
P\{d(k)>T a\} \geq \lambda_{T a}
$$

If the equation (5) is true, that means there's a probability of $\left(1-\lambda_{T a}\right)$ that target has made a strong maneuver. In order to guarantee the tracking accuracy and that the model can still adapt to target's maneuvering, $\alpha(k)$ and $a_{ \pm \max }$ should be improved at the same time.

If not, it illustrate that the target moves without or with just very week maneuvering, in which case tracking performance can be remained without changing $\alpha(k)$ or $a_{ \pm \max }$.

\subsection{Adaptive Parameters Algorithm}

Define the calculation of the average acceleration of target as:

$$
\bar{a}_{p}=\frac{1}{l} \sum_{i=k-l}^{k-1} \ddot{x}(i)
$$

Where $l$ stands for step length, the recommended values of which usually range from 3 to $10, \ddot{x}(i)$ is the estimate of target's acceleration at the moment of $i, \bar{a}_{p}$ is a fixed constant when $k \leq l$.

When equation (5) is true, i.e. the target's maneuver is detected to be strong, in 3.1 , the model parameters should be updated as:

$$
\sigma_{a}^{2}= \begin{cases}\frac{4-\pi}{\pi}\left[\sqrt{d(k)-2} a_{\max }-\bar{a}_{p}(k)\right]^{2} & \bar{a}_{p}(k) \geq 0 \\ \frac{4-\pi}{\pi}\left[\sqrt{d(k)-2}+\bar{a}_{p}(k)\right]^{2} & \bar{a}_{p}(k)<0\end{cases}
$$




$$
\alpha=\sqrt{d(k)-2} \alpha_{0}
$$

Therefore, the parameters of CS model can be adaptive according to equation (7) (8), and then match the real maneuvering of target, and finally improve the target tracking accuracy.

\section{Filtering Algorithm of CL Model}

\subsection{Model of Target Motion}

Assume that the target is making curve motion in the X-Y plane at the moment of $k$ with the state vector, centripetal acceleration and normal acceleration respectively of $\left[\begin{array}{llllll}x & \dot{x} & \ddot{x} & y & \dot{y} & \ddot{y}\end{array}\right]^{T}, a_{n}$ and $a_{t}$. While $\phi$ is the angle between the direction of velocity and north (clockwise stands for positive), $R$ presents turning radius. The relationships of variables mentioned above are as follow:

$$
\begin{gathered}
v(k)=R \square_{W(k)} \\
a_{t}(k)=\left.\frac{d v(t)}{d t}\right|_{t=k}=R \square \beta(k) \\
a_{n}=R \square_{W}(k)^{2}=v(k) \square_{W}(k)
\end{gathered}
$$

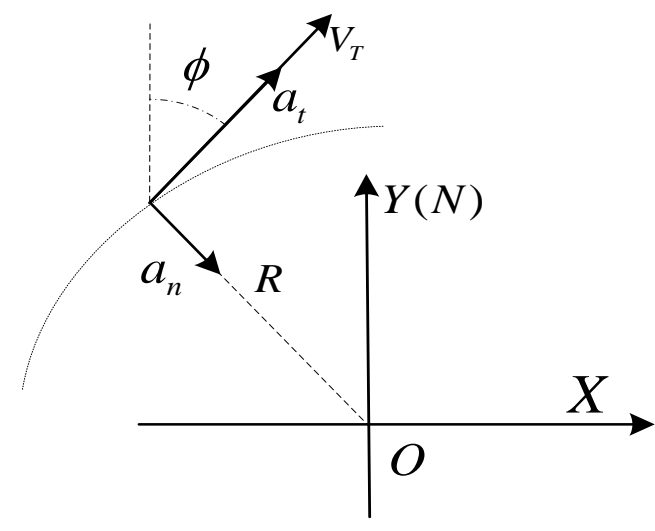

\section{Figure 2. X-Y Plane Graph of Target Curve Motion}

Where $w(k), \beta(k)$ stands for angular velocity and angular acceleration of the target respectively.

According to Figure 2, the projection of velocity in both $\mathrm{X}$ and $\mathrm{Y}$ direction are:

$$
\begin{gathered}
\dot{x}(t)=v(t) \square \sin (\phi(t)) \\
\dot{y}(t)=v(t) \square \cos (\phi(t))
\end{gathered}
$$

The second-order continuous differential state equation of the target can be deduced from equations (12) (13) as: 


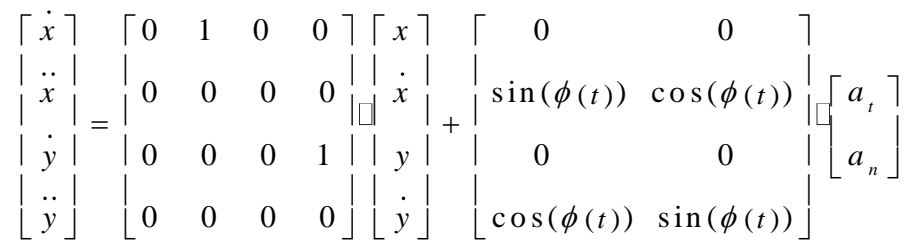

There are two conditions (15) (16) hold for modern vehicles when the onboard radar keeps a normal scan frequency.

$$
\begin{gathered}
\left|\frac{\dot{a}_{n}(k)}{a_{n}(k)}\right|<\left|\frac{a_{t}(k)}{v(k)}\right| \\
\left|\frac{a_{t}(k)}{v(k)}\right|<\frac{2}{T}
\end{gathered}
$$

Where $k$ and $T$ stands for sampling instant and sampling period.

The following equation (17) is the discrete form of the integration of equation (14) according to (13) (14) under the conditions of (15) (16):

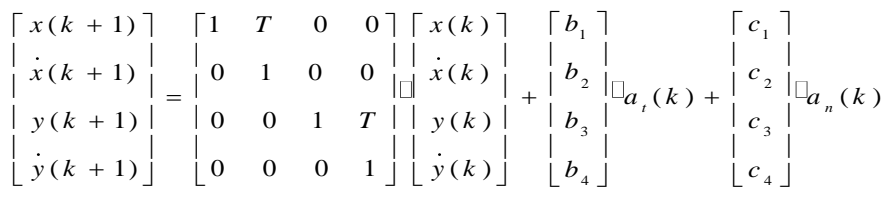

Where:

$$
\begin{aligned}
& b_{1}=\frac{\sin (\phi(k))}{w(k)^{2}}-\frac{\sin (\phi(k)+T w(k))}{w(k)^{2}}+\frac{T \cos (\phi(k))}{w(k)} \\
& b_{2}=\frac{\cos (\phi(k))}{w(k)}-\frac{\cos (\phi(k)+T w(k))}{w(k)} \\
& b_{3}=\frac{\cos (\phi(k))}{w(k)^{2}}-\frac{\cos (\phi(k)+T w(k))}{w(k)^{2}}-\frac{T \sin (\phi(k))}{w(k)} \\
& b_{4}=-\frac{\sin (\phi(k))}{w(k)}+\frac{\sin (\phi(k)+T w(k))}{w(k)} \\
& c_{1}=\frac{\cos (\phi(k))}{w(k)^{2}}-\frac{\cos (\phi(k)+T w(k))}{w(k)^{2}}-\frac{T \sin (\phi(k))}{w(k)} \\
& c_{2}=-\frac{\sin (\phi(k))}{w(k)}+\frac{\sin (\phi(k)+T w(k))}{w(k)} \\
& c_{4}=-\frac{\cos (\phi(k))}{w(k)}+\frac{\cos (\phi(k)+T w(k))}{w(k)} \\
& c_{3}=-\frac{\sin (\phi(k))}{w(k)^{2}}+\frac{\sin (\phi(k)+T w(k))}{w(k)^{2}}-\frac{T \cos (\phi(k))}{w(k)}
\end{aligned}
$$




$$
\phi(k)=\arctan \left(\frac{\dot{x}(k)}{\dot{y}(k)}\right)
$$

Equation (17) indicates that:

when $a_{t}(k)=0, a_{n}(k)=0$, it represents CV model;

when $a_{t}(k) \neq 0, a_{n}(k)=0$, it represents CA model;

when $a_{t}(k)=0, a_{n}(k) \neq 0$, it represents CT model.

With equations (11) (13), the third item on the right-hand side of equation (17) can be transformed into the form of $x(k), \dot{x}(k), y(k), \dot{y}(k)$ and thus can be simplified as:

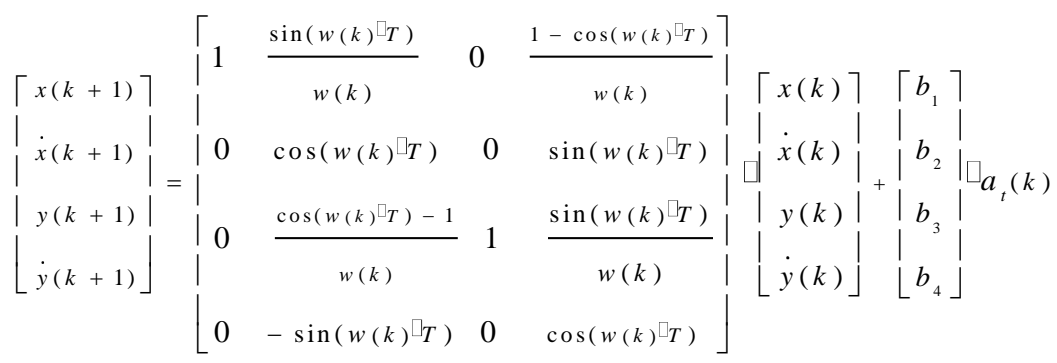

\subsection{Filtering Algorithm of CL Model}

The equation (27) indicates that the values of angular velocity $w(t)$ and normal angular acceleration $a_{t}(k)$ need to be updated in real time. Considering that the improved CS model can provide the estimate of $a_{t}(k)$, we designed a estimate algorithm (28) for angular velocity $w(t)$ based on a time varying model to avoid adding an external model to do the task.

$$
\begin{gathered}
w(k+1)=w(k)+\frac{w(k) T}{v(k)} \square_{a_{t}}(k) \cong w(k)+\frac{\Delta \phi(k)}{v(k)} \square_{a_{t}}(k) \\
\Delta \phi(k)=\phi(k)-\phi(k-1) \\
v(k)=\sqrt{\dot{x}(k)^{2}+\dot{y}(k)^{2}}
\end{gathered}
$$

With equation (26), (29) and (30), equation (28) can be transformed into (31):

$$
w(k+1)=w(k)+\frac{\arctan \left(\frac{\dot{x}(k)}{\dot{y}(k)}\right)-\arctan \left(\frac{\dot{x}(k-1)}{\dot{y}(k-1)}\right)}{\sqrt{\dot{x}(k)^{2}+\dot{y}(k)^{2}}} a_{t}(k)
$$

The dimension of the state variables in equation (27) can be expanded by equation (31) and substituted into $w(k)$ to make:

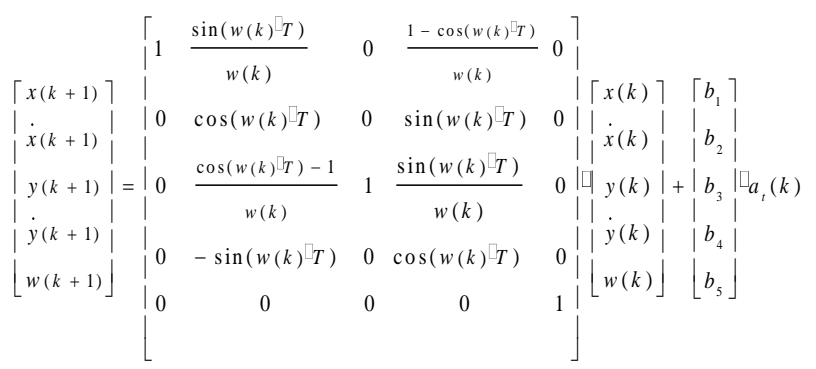


Where:

$$
b_{5}=\frac{\arctan \left(\frac{\dot{x}(k)}{\dot{y}(k)}\right)-\arctan \left(\frac{\dot{x}(k-1)}{\dot{y}(k-1)}\right)}{\sqrt{\dot{x}(k)^{2}+\dot{y}(k)^{2}}}
$$

By updating the values of $a_{t}(k)$ and $w(t)$ in real time using algorithms in Section 3 and equation (32) respectively, target tracking is realized.

\section{Simulation Results}

\subsection{The Simulation Results of Acceleration Estimate in ICS Model}

Simulation Scenario: Assume that the target's maneuvers are limited in a horizontal plan. The initial state of target is $X(0)=\left[x_{0}, \dot{x}_{0}, \ddot{x}_{0}, y_{0}, \dot{y}_{0}, \ddot{y}_{0}\right]^{T}=[1000,120,0,2000,100,0]^{T}$,

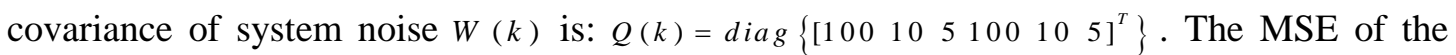
measurement noise (two-coordinate radar) is: $\sigma_{x y}=\operatorname{diag}[50,50]^{T}$, i.e., covariance of measurement noise $V(k)$ is: $R(k)=\operatorname{diag}[50,50]^{T} \mathrm{~m}$. The sample period and total simulation time is $T=0.1 \mathrm{~s}$ and 50s respectively. The maneuvers of target are listed in Table 1.

Table 1. Maneuvers of Target

\begin{tabular}{ccc}
\hline Time & Maneuver Type & Acceleration $\left[a_{x}, a_{y}\right]$ \\
\hline $0 \sim 10 \mathrm{~s}$ & $\mathrm{CV}($ Constant Velocity) & {$[0,0]$} \\
$10 \sim 15 \mathrm{~s}$ & $\mathrm{CA}($ Constant Acceleration) & {$[20,-20]$} \\
$15 \sim 30 \mathrm{~s}$ & $\mathrm{CV}$ & {$[0,0]$} \\
$30 \sim 33 \mathrm{~s}$ & $\mathrm{CA}$ & {$[-20,20]$} \\
$33 \sim 50 \mathrm{~s}$ & $\mathrm{CV}$ & {$[0,0]$} \\
\hline
\end{tabular}

The estimate results of acceleration are shown in Figure 3 5:

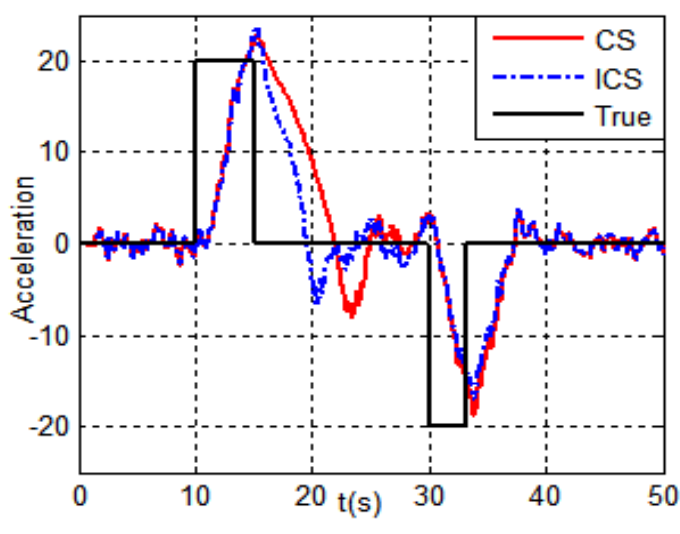

Figure 3. Acceleration in $\mathrm{X}$ Direction

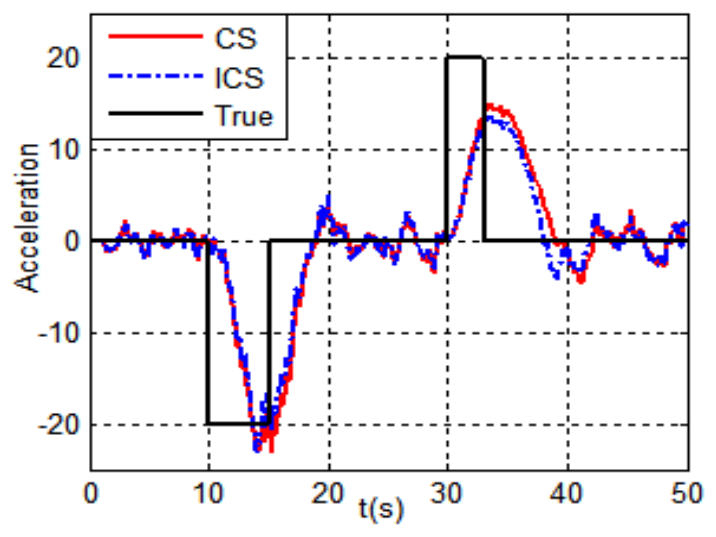

Figure 4. Acceleration in Y Direction 
Simulation results in Figure 3-4 show that both of CS model and ICS model would generate delay when estimating the acceleration of target. For example, when $t=10 \mathrm{~s}$ the target changes from CV motion into CA motion with acceleration of $\left[a_{x}, a_{y}\right]=[20,-20] \mathrm{m} / \mathrm{s}^{2}$. But their acceleration estimates cannot adapt to target's real acceleration until $t=15 \mathrm{~s}$, that's because the acceleration estimates of both CS and ICS model depend on the variance of estimate error $Q(k)$, which changes with time delay, thus delaying the correction of estimate.

However, Figure 3-4 illustrate that ICS model takes less time than CS model to adjust its parameters to adapt to the true acceleration. For example, when target returns to $\mathrm{CV}$ motion at time $t=15 \mathrm{~s}$, ICS model tracks on the true acceleration close to $t=23 \mathrm{~s}$, but CS model does not catch up until $t=27 \mathrm{~s}$. At the same time, the ICS model has less overshoot than CS model, which indicates that the former conduct more stable tracking performance than the latter.

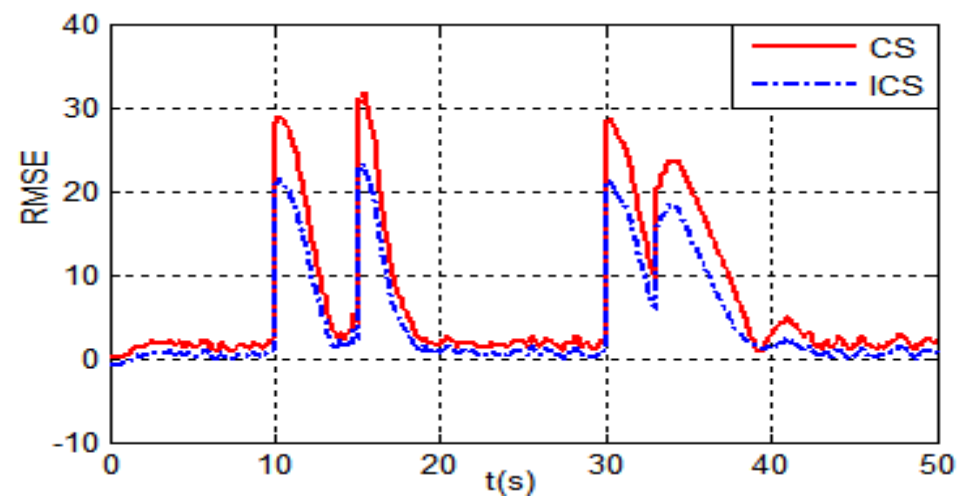

Figure 5. Acceleration Estimate RMSE (N=50)

As shown in Figure 5, the 50 sets of Monte Carlo simulation results show that the estimate RMSE (Root Mean Squared Error) of ICS model is much smaller than that of CS model. The average RMSE shown in Table 2 demonstrates that the tracking accuracy of ICS model improved by $35.81 \%$ than that of CS model.

Table 2. Average RMSE of Acceleration Estimate

\begin{tabular}{cccc}
\hline RMSE & CS & ICS & Precision Improved \\
\hline Acceleration Estimate & 6.87 & 4.41 & $35.81 \%$ \\
\hline
\end{tabular}

\subsection{The Two-Layer Nested Model Simulation Results}

With the same simulation scenario in 5.1, target tracking results by ICS and CS-CL model are shown in Figure 6 11. 


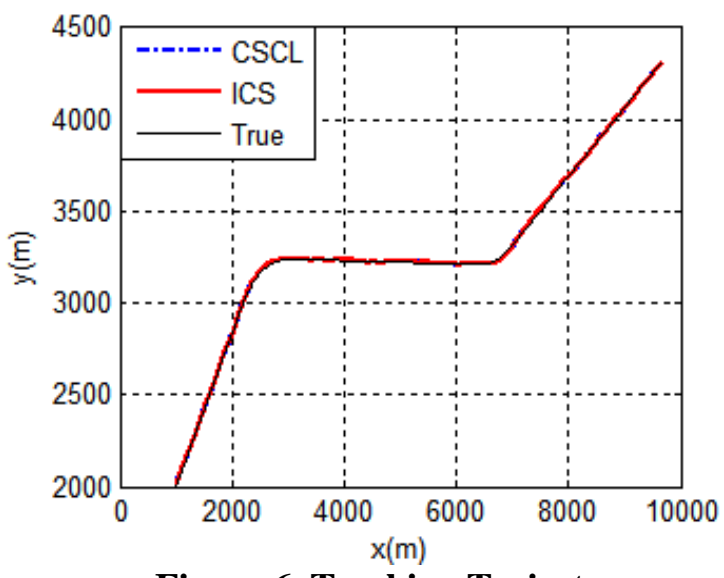

Figure 6. Tracking Trajectory

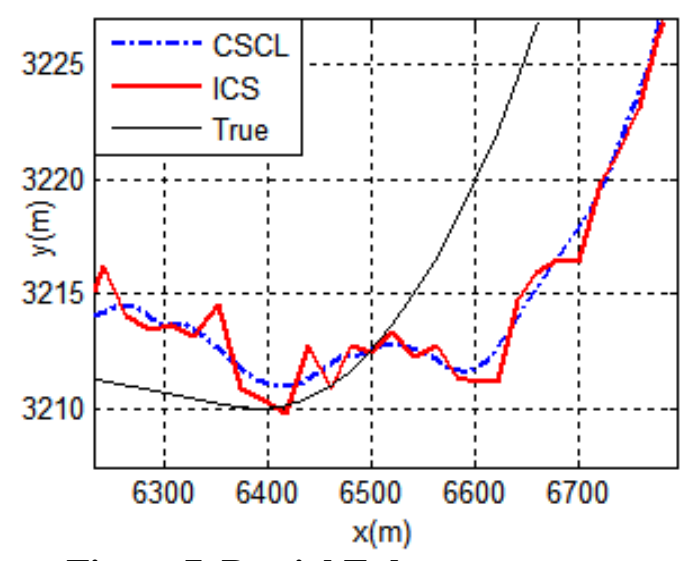

Figure 7. Partial Enlargement View of Figure 6

Figure 6 shows that CSCL and ICS model can conduct similar extent of accuracy in target's tracking trajectory, but the partial enlargement view of Figure 6, as shown in Figure 7, shows that when target maneuvers with a large angular velocity, tracking results of CS-CL model is obviously smoother and closer to the true trajectory than ICS.

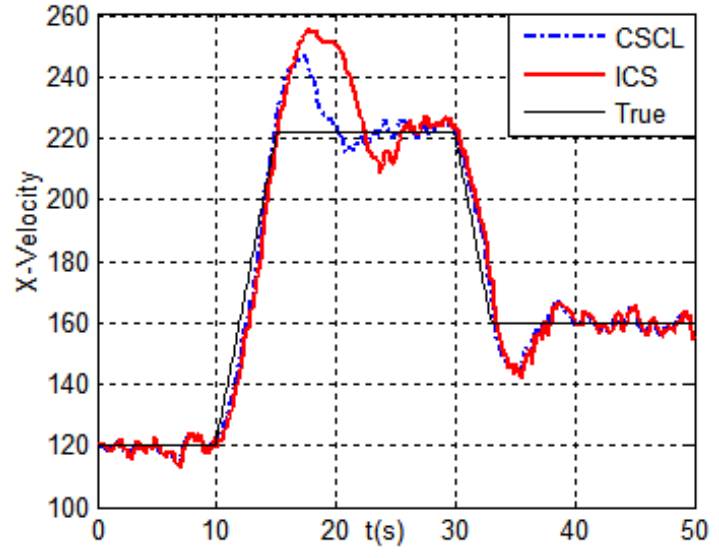

Figure 8. Velocity Estimate in $\mathrm{X}$ direction

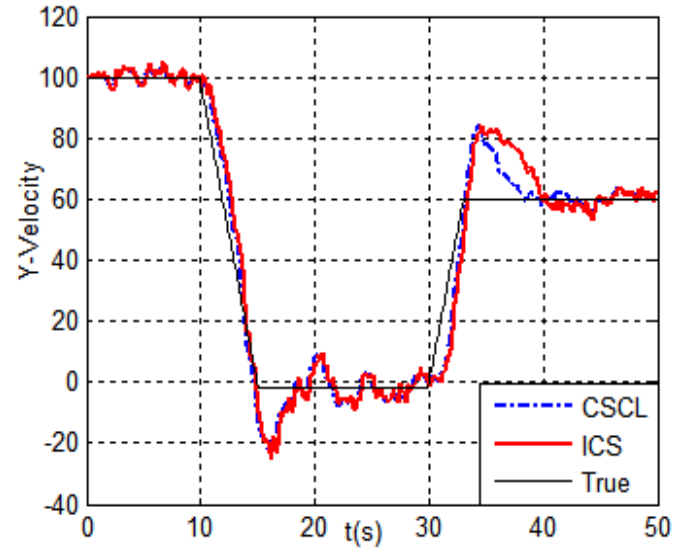

Figure 9. Velocity Estimate in Y direction

The estimate of velocity in Figure 8 shows that when target returns to CV motion from CA motion at $t=15 \mathrm{~s}, \mathrm{CS}-\mathrm{CL}$ and ICS model can approximately adapt to that at $t=22 \mathrm{~s}$ and $26 s$ respectively, i.e., the time delay is 7s by CS-CL model and 11s by ICS model. Similarly, as shown in Figure 9, when target returns to CV motion from CA motion at $t=33 \mathrm{~s}$, CS-CL model would produce a good tracking performance from $t=35 \mathrm{~s}$, but ICS at $t=38 \mathrm{~s}$. Results in Figure 8 9 indicate that CS-CL model conducts better dynamic performance than ICS model. 


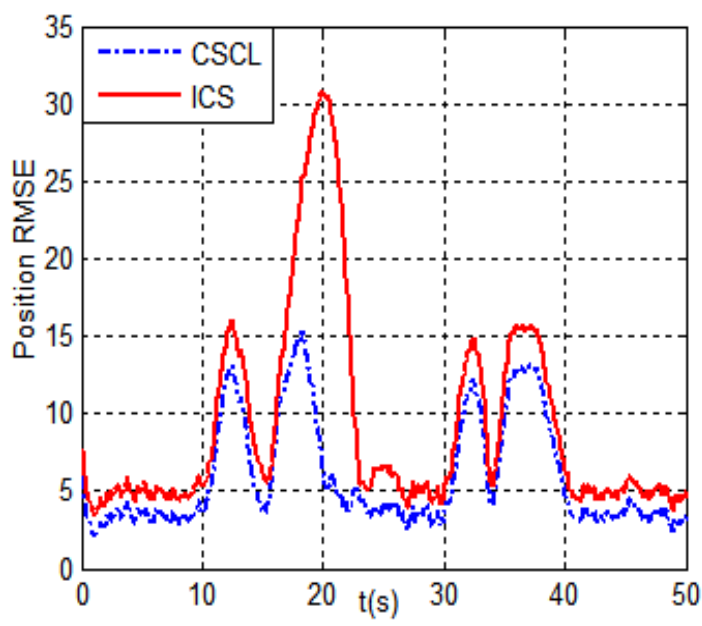

Figure 10. Position Estimate RMSE (N=50)

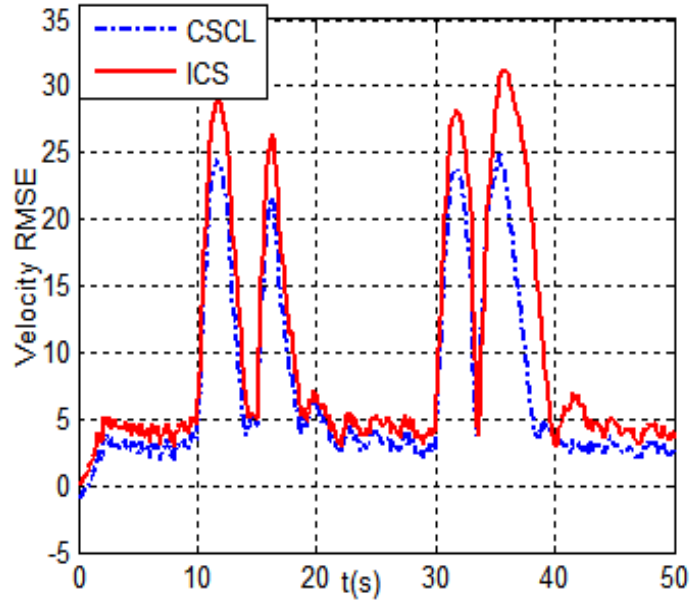

Figure 11. Velocity Estimate RMSE $(\mathbf{N}=\mathbf{5 0})$

As shown in Figure 10 11, the 50 sets of Monte Carlo simulation results show that CS-CL model can conduct more accurate target tracking performance than ICS model, particularly when target makes strong maneuver during $10 \sim 23 \mathrm{~s}$. Results in Table 2 further illustrate that CS-CL model makes the tracking precision in position and velocity improved by $36.22 \%$ and $58.38 \%$ respectively than ICS model.

Table 2. Average RMSE of Position \& Velocity Estimate

\begin{tabular}{cccc}
\hline RMSE & ICS & CSCL & Precision Improved \\
\hline Position Estimate & 9.28 & 5.92 & $36.22 \%$ \\
Velocity Estimate & 9.72 & 6.13 & $58.38 \%$ \\
\hline
\end{tabular}

\section{Conclusions}

An improved CS (ICS) model is presented straightforwardly, which can simultaneously accommodate varying angular velocity of target in its possible maneuvering behavior. CL model is subsequently presented for its effectiveness in adjusting to target motion. Using ICS as inner model and CL model as outer model, a two-layer nested model is constructed. Based on this nested model, a novel algorithm is proposed to ameliorate tracking performance of strong maneuvering target. Simulation results validate the effectiveness of this novel algorithm.

Note that the success of the novel algorithm is mainly in tracking accuracy and stability, the development of the methodology to reduce time delay to achieve better efficiency can be involved into further research.

\section{Acknowledgments}

This work is supported by Natural Science Foundation of Chongqing (cstc2013jcyjA30015) and Special Research Plan of Education Department of Shaanxi Provincial Government (2013JK0219). 


\section{References}

[1] X. R. Li and V. P. Jilkov, "Survey of maneuvering target tracking", Part I. Dynamic models, Aerospace and Electronic Systems, IEEE Transactions, vol. 39, no. 4, (2003), pp. 1333-1364.

[2] Y. Bar-Shalom, P. K. Willett and X. Tian, "Tracking and data fusion", A Handbook of Algorithms, Yaakov Bar-Shalom, (2011).

[3] E. Xu, Z. Ding and S. Dasgupta, "Target tracking and mobile sensor navigation in wireless sensor networks", Mobile Computing, IEEE Transactions, vol. 12, no. 1, (2013), pp. 177-186.

[4] W. Yang, Y. Fu and X. Li, "Joint target tracking and classification via RFS-based multiple model filtering", Information Fusion, vol. 18, (2014), pp. 101-106.

[5] H. Wei-ping, X. Yu and W. Jie, "A nonlinear maneuver-tracking algorithm based on modified current statistical model", Control Theory \& Applications, vol. 28, no. 12, (2012), pp. 1723-1728.

[6] Y. J. Zhang and Z. Geng, "Detection of Target Maneuver from Bearings-Only Measurements", Aerospace and Electronic Systems, IEEE Transactions, vol. 49, no. 3, (2013), pp. 2028-2034.

[7] Y. Jin-long, J. Hong-bing and L. Juan-li, "Adaptive curvilinear model based target tracking algorithm for multiple passive sensors", Control and Decision, vol. 26, no. 8, (2011), pp. 1126-1130.

[8] S. Zhao, F. Lin and K. Peng, "Distributed control of angle-constrained cyclic formations using bearing-only measurements", Systems \& Control Letters, vol. 63, (2014), pp. 12-24.

[9] B. Grocholsky, "Information-theoretic control of multiple sensor platforms", (2006).

[10] X. Yuan, F. Lian and C. Han, "Models and Algorithms for Tracking Target with Coordinated Turn Motion", Mathematical Problems in Engineering, (2014).

[11] S. Sadhu, S. Mondal and M. Srinivasan, "Sigma point Kalman filter for bearing only tracking", Signal processing, vol. 86, no. 12, (2006), pp. 3769-3777.

[12] C. L. Wang, T. M. Wang and J. H. Liang, "Bearing-only visual SLAM for small unmanned aerial vehicles in GPS-denied environments", International Journal of Automation and Computing, vol. 10, no. 5, (2013), pp. 387-396.

[13] H. Choi and Y. Kim, "UAV guidance using a monocular-vision sensor for aerial target tracking", Control Engineering Practice, vol. 22, (2014), pp. 10-19.

[14] Y. J. Zhang and G. Z. Xu, "Bearings-only target motion analysis via instrumental variable estimation", Signal Processing, IEEE Transactions on, vol. 58, no. 11, (2010), pp. 5523-5533.

[15] H. S. Ramos, A. Boukerche and R. W. Pazzi, "Cooperative target tracking in vehicular sensor networks", Wireless Communications, IEEE, vol. 19, no. 5, (2012), pp. 66-73.

\section{Authors}

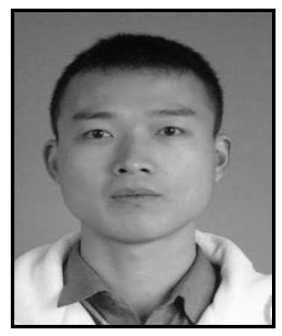

Jinshuan Peng, Male, Doctor of Engineering, Associate Professor. He received his Ph.D. in vehicle operation engineering (2012) from Chang'an university. Now he is an assistant professor of transportation, Chongqing Jiaotong University. His current research interests include driving behavior and E-commerce implementation evaluation.

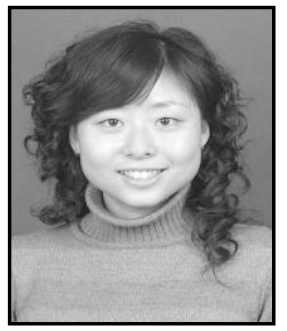

Lei Xu, Female, Doctor of Logistic Engineering, Associate Professor. She received her Ph.D. in logistic engineering (2011) from Chang'an university. Now she is an assistant professor of traffic management, Chongqing Jiaotong University. Her current research interests include Ecommerce implementation evaluation and supply chain emergency management. 


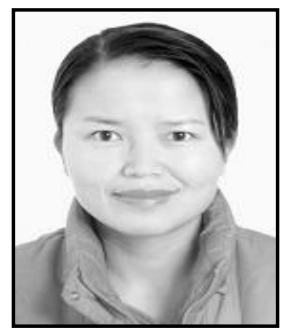

Liping Wang, Female, Master of Engineering, Associate Professor. She received the B. Eng. degree in mechanical engineering from Beihang University, Beijing, China, in 1996 and the M. Eng. degree in mechanical engineering from Tianjin University, Tianjin, China, in 2005.

She is an Associate Professor in Shanghai Jianqiao University, Shanghai, China. Her research interests include mechanical design, mechanical processing, reverse engineering, biomechanics and biomaterials.

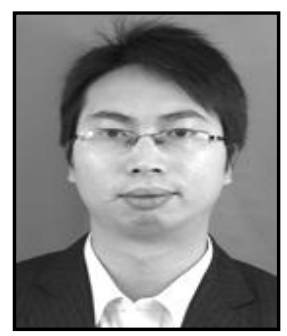

Xiaoxiang Zhou, Male, Master of Logistic Engineering, Associate Professor. He received his master degree in logistic management (2011) from China University of Mining Technology. Now he is an assistant professor of logistics management, Chongqing Jiaotong University. His current research interests include logistics system evaluation and human resource management. 